FTUAM 99-8

UG-FT-97/99

hep-ph/9904344

April 14, 1999

(Revised August, 11, 1999)

\title{
Calculation of Electroproduction to NNLO and Precision Determination of $\alpha_{s}$
}

\author{
J. Santiago \\ Departamento de Física Teórica \\ y del Cosmos, \\ Universidad de Granada, \\ E-18071, Granada, Spain. \\ and \\ F. J. Ynduráin \\ Departamento de Física Teórica, C-XI, \\ Universidad Autónoma de Madrid, \\ Canto Blanco, \\ E-28049, Madrid, Spain.
}

\begin{abstract}
We use the known values of the two loop Wilson coefficients and the three loop anomalous dimension matrix $\gamma(n)$ to perform a next-to-next-to leading order (NNLO) calculation of $e p$ deep inelastic scattering. Because $\gamma(n)$ is only known for a few values of $n$, the method of average reconstruction has to be used, which leaves 102 effective experimental points, for 12 parameters: the QCD mass $\Lambda$, and 11 initial values for the moments of the structure functions. The data points spread in the range of momenta $2.5 \mathrm{GeV}^{2} \leq Q^{2} \leq 230 \mathrm{GeV}^{2}$.

The $\chi^{2} /$ d.o.f. decreases substantially when going from LO to NLO, and also from NLO to NNLO (although only a little now) to $\chi^{2} /$ d.o.f. $=79.2 /(102-$ 12). The favoured value of $\Lambda$ is

$$
\Lambda\left(n_{f}=4,3 \text { loop }\right)=282.7 \pm 35.1 \mathrm{MeV},
$$

corresponding to the value of the coupling at the $\mathrm{Z}$ mass of

$$
\alpha_{s}^{(3 \text { loop })}\left(M_{Z}^{2}\right)=0.1172 \pm 0.0024
$$

The calculation, which constitutes a very precise test of QCD, includes target mass corrections; the error takes into account experimental errors and higher twist effects among other estimated theoretical errors.
\end{abstract}


Typeset with $\mathrm{P}_{\mathrm{H}}{ }^{\mathrm{M}}{ }^{\mathrm{Ma}} \mathrm{T}_{\mathrm{E}} \mathrm{X}$ 
-CALCUlATION OF ELECTROPRODUCTION TO NNLO AND PRECISION DETERMINATION OF $\alpha_{s}{ }^{-}$

\section{Introduction}

Deep inelastic scattering (DIS), in particular of electrons (or muons) on protons, constituted one of the first probes of hadron structure. The calculation of QCD-induced scaling violations in the structure functions ${ }^{[1]}$ yielded some of the earliest qualitative checks of the quark-gluon theory of hadron interactions, as well as providing the first two loop determinations of the strong coupling constant ${ }^{[2]}$. In this last ref. 2 , the two loop results for the nonsinglet anomalous dimensions ${ }^{[2,3]}$ were used, together with the one loop Wilson coefficients $^{\left[{ }^{[}\right]}$to get a calculation of the nonsinglet structure functions to next to leading order (NLO). ${ }^{1}$ Because both anomalous dimensions and coefficient functions were known completely, it was possible to use a method devised by Gross ${ }^{[5]}$ to LO, generalized in ref. 2 to NLO, to obtain an exact, pointlike (in $x$ ) reconstruction of the nonsinglet part of $F_{2}\left(x, Q^{2}\right)$, and of $x F_{3}\left(x, Q^{2}\right)$.

Since then the calculations have been extended to include singlet anomalous dimensions (ref. 6; see ref. 7 for a collection of two loop formulas). Furthermore, the two loop Wilson coefficients are also known ${ }^{[8]}$ so that, to perform a NNLO calculation, only the three loop anomalous dimensions are needed. There does not exist at present a full calculation of these; but a partial computation has been made available recently. ${ }^{[9]}$ This has been used in some papers (see e.g. ref. 10 and work quoted there) to perform an evaluation of deep inelastic structure functions to next-to-next-to-leading order, NNLO. These evaluations, however, present a number of shortcomings. First of all, the values of the moments which are not known are found by interpolation of the known moments; and likewise the experimental values of the moments, with which to compare the theory, are obtained also interpolating and extrapolating experimental data. These procedures are dangerous in that they imply unknown systematic errors, thus putting in jeopardy an already delicate calculation (NNLO effects are in themselves small, generally speaking). Perhaps more important, only nonsinglet structure functions (specifically, $x F_{3}$ for $\nu$ scattering in ref. 10) were considered: while the best data exist for the $e p$ (or $\mu p$ ) structure function $F_{2}$, with a strong singlet component.

In the present paper we improve on this in the following ways. First of all, we extend the calculation to include singlet as well as nonsinglet structure functions. This allows us to use both the very precise, comparatively low energy, electroproduction SLAC data, and the recent, very high energy HERA results, plus some intermediate energy muon-production data. This provides a huge range of $Q^{2}$ values. Secondly, we employ the method of Bernstein polynomials. This method was developed in ref. 11 for calculating averages of the structure functions around values of $x$ where experimental data are available, in terms of only a finite set of known moments: thus at the same time avoiding the problem of interpolating the theoretical values of the anomalous dimension, and greatly diminishing the errors inherent to the calculation of the experimental input, as only known sets of data points are essentially relevant.

In this way we find a precise comparison of electroproduction DIS data with theory, obtaining in particular a very accurate evaluation of the QCD coupling and mass. With respect to the last we find

$$
\begin{aligned}
\alpha_{s}^{(3 \text { loop })}\left(M_{Z}^{2}\right) & =0.1172 \pm 0.0017(\text { statistical }) \pm 0.0017 \text { (systematic) } \\
& =0.1172 \pm 0.0024,
\end{aligned}
$$

to which corresponds the value and error of the mass parameter

$$
\Lambda\left(n_{f}=4,3 \text { loop }\right)=283 \pm 35 \mathrm{MeV} .
$$

\footnotetext{
${ }^{1}$ To leading order (LO) one requires the one loop anomalous dimension and the tree level Wilson coefficients. Likewise, a NLO calculation uses one loop Wilson coefficients and two loop anomalous dimension and the NNLO one (which is the subject of the present paper) implies two loop Wilson coefficients and three loop anomalous dimensions.
} 


\section{The evolution equations}

To describe the calculation, let us establish some notation first. We split $F_{2}$ into a singlet and a nonsinglet part,

$$
F_{2}\left(x, Q^{2}\right)=F_{S}\left(x, Q^{2}\right)+F_{N S}\left(x, Q^{2}\right) .
$$

We also define the gluon structure function, $F_{G}\left(x, Q^{2}\right) \equiv x G\left(x, Q^{2}\right), G$ being the gluon density. Finally, we form the vector with components $F_{S}, F_{G}$ :

$$
F\left(x, Q^{2}\right)=\left(\begin{array}{c}
F_{S}\left(x, Q^{2}\right) \\
F_{G}\left(x, Q^{2}\right)
\end{array}\right)
$$

We obtain the moments by projecting these with powers of $x$ :

$$
\begin{aligned}
\mu_{N S}\left(n ; Q^{2}\right) & =\int_{0}^{1} \mathrm{~d} x x^{n-2} F_{N S}\left(x, Q^{2}\right) \\
\mu_{i}\left(n ; Q^{2}\right) & =\int_{0}^{1} \mathrm{~d} x x^{n-2} F_{i}\left(x, Q^{2}\right) ; \quad i=S, G .
\end{aligned}
$$

If we write a light cone expansion for the moments, this is given in terms of the Wilson coefficients and the anomalous dimensions of appropriate sets of operators (for details on this, see any text on QCD, e.g., ref. 12). Let $t=\frac{1}{2} \log \left(Q^{2} / \nu^{2}\right)$, with $\nu$ the renormalization scale, and $a=\alpha_{s}\left(Q^{2}\right) / 4 \pi, a^{\prime}=\alpha_{s}\left(Q^{\prime 2}\right) / 4 \pi$. For the nonsinglet component, we have

$$
\mu_{N S}\left(n ; Q^{2}\right)=C_{N S}\left(n, \alpha_{s}\left(Q^{2}\right) / 4 \pi\right) \exp \left[-\int_{0}^{t} \mathrm{~d} t^{\prime} \gamma_{N S}\left(a^{\prime}\right)\right] A_{N S}\left(\nu^{2}\right)
$$

whereas the singlet equations are of a matrix character,

$$
\mu_{i}\left(n ; Q^{2}\right)=\sum_{j} C_{j}\left(n, \alpha_{s}\left(Q^{2}\right) / 4 \pi\right)\left\{T \exp \left[-\int_{0}^{t} \mathrm{~d} t^{\prime} \gamma\left(a^{\prime}\right)\right] A\left(\nu^{2}\right)\right\}_{j i}
$$

The $\gamma_{N S}, \gamma$ are the NS, S anomalous dimension and anomalous dimension matrix, and the $A_{N S}, A$ unknown matrix elements of certain operators. What the calculations of Larin et al. ${ }^{[9]}$ provide is the expression of the gammas to three loops (third order in $\alpha_{s}$ ) for $n=2,4,6,8 .^{2}$

Before discussing how to use this information, let us write the equations that follow from Eqs. (2.3). We will consider in some detail only the singlet component; the NS one is fairly trivial. We start by completing a two by two matrix $C$ from $C_{S}, C_{G}$,

$$
C=\left(\begin{array}{ll}
C_{S} & C_{12} \\
C_{G} & C_{22}
\end{array}\right) \equiv\left(\begin{array}{ll}
C_{11} & C_{12} \\
C_{21} & C_{22}
\end{array}\right)
$$

by requiring it to commute with the anomalous dimension matrix:

$$
[C, \gamma]=0
$$

(the index $n$ will be omitted in some of the formulas to lighten the notation). We define

$$
D(a)=\frac{\gamma(a)}{2 \beta(a)}
$$

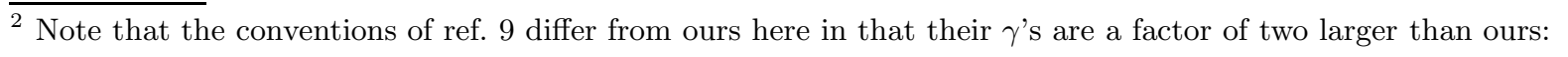

$$
2 \gamma^{\text {ref. } 9}(n)=\gamma^{\text {here }}(n)
$$


and write the series expansions,

$$
\begin{gathered}
C(a)=1+C^{(1)} a+C^{(2)} a^{2}+\ldots \\
\gamma(a)=\gamma^{(0)} a+\gamma^{(1)} a^{2}+\gamma^{(2)} a^{3}+\ldots \\
-\beta(a)=\beta_{0} a^{2}+\beta_{1} a^{3}+\beta_{2} a^{4}+\ldots, \\
D(a)=\frac{1}{a} D^{(0)}+D^{(1)}+D^{(2)} a+\ldots ; \\
D^{(0)}=\frac{-1}{2 \beta_{0}} \gamma^{(0)} ; D^{(1)}=\frac{-1}{2 \beta_{0}}\left(\gamma^{(1)}-\frac{\beta_{1}}{\beta_{0}} \gamma^{(0)}\right) ; \\
D^{(2)}=\frac{-1}{2 \beta_{0}}\left[\gamma^{(2)}-\frac{\beta_{1}}{\beta_{0}} \gamma^{(1)}+\left(\frac{\beta_{1}^{2}}{\beta_{0}}-\frac{\beta_{2}}{\beta_{0}}\right) \gamma^{(0)}\right] ; \cdots .
\end{gathered}
$$

The commutativity of $C$ and $\gamma$, order by order in perturbation theory, implies the equations

$$
\begin{aligned}
& C_{21}^{(1)}=\frac{\gamma_{21}^{(0)}}{\gamma_{12}^{(0)}} C_{12}^{(1)}, \\
& C_{22}^{(1)}=C_{11}^{(1)}+\frac{\gamma_{22}^{(0)}-\gamma_{11}^{(0)}}{\gamma_{12}^{(0)}} C_{12}^{(1)} ; \\
& C_{21}^{(2)}=\frac{C_{12}^{(1)} \gamma_{21}^{(1)}+C_{12}^{(2)} \gamma_{21}^{(0)}-C_{21}^{(1)} \gamma_{12}^{(1)}}{\gamma_{12}^{(0)}}, \\
& C_{22}^{(2)}=C_{11}^{(2)}+\frac{\gamma_{22}^{(0)}-\gamma_{11}^{(0)}}{\gamma_{12}^{(0)}} C_{12}^{(2)}+\frac{\gamma_{22}^{(1)}-\gamma_{11}^{(1)}}{\gamma_{12}^{(0)}} C_{12}^{(1)}+\frac{C_{11}^{(1)}-C_{22}^{(1)}}{\gamma_{12}^{(0)}} \gamma_{12}^{(1)} .
\end{aligned}
$$

These equations allow us to find the $C_{21}, C_{22}$ in terms of the $C_{11}, C_{12}$ and the $\gamma_{i j}$ which in turn are taken from ref. 9.

For the moments equations, we define the matrix $S$ that diagonalizes the LO anomalous dimension matrix, so that

We take it to be

$$
S^{-1} D^{(0)} S=\hat{D}^{(0)}=\left(\begin{array}{cc}
d_{+} & 0 \\
0 & d_{-}
\end{array}\right), d_{+}>d_{-}
$$

and we define also

$$
S=\left(\begin{array}{cc}
1 & \frac{D_{12}^{(0)}}{d_{-}-d_{+}} \\
\frac{d_{+}-D_{11}^{(0)}}{D_{12}^{(0)}} & \frac{d_{-}-D_{11}^{(0)}}{d_{-}-d_{+}}
\end{array}\right), S_{11}=\operatorname{det} S=1,
$$

$$
\begin{array}{r}
S^{-1} D^{(N)} S \equiv \bar{D}^{(N)}, S^{-1} M^{(N)} S \equiv \bar{M}^{(N)}, S^{-1} \gamma^{(N)} S \equiv \bar{\gamma}^{(N)} \quad \text { etc. } \\
\left(\bar{D}^{(0)}=\hat{D}^{(0)}\right) .
\end{array}
$$

Here the matrix $M$ is defined to be such that

$$
\frac{\partial}{\partial a}\left\{a^{D^{(0)}} M(a) C^{-1}(a) \mu(a)\right\}=0,
$$

and it has the series expansion

$$
M(a)=1+M^{(1)} a+M^{(2)} a^{2}+\cdots .
$$

From Eqs. (2.8c), (2.9) we find the explicit values of the $M$ :

$$
\bar{M}^{(1)}=\left(\begin{array}{cc}
\bar{D}_{11}^{(1)} & \frac{1}{1+d_{+}-d_{-}} \bar{D}_{12}^{(1)} \\
\frac{1}{1+d_{-}-d_{+}} \bar{D}_{21}^{(1)} & \bar{D}_{22}^{(1)}
\end{array}\right)
$$


-J. SANTIAGO AND F. J. YNDURÁIN-

$$
\bar{M}^{(2)}=\left(\begin{array}{cc}
\frac{1}{2}\left[\bar{D}_{11}^{(2)}+\left(\bar{M}^{(1)} \bar{D}^{(1)}\right)_{11}\right] & \frac{1}{2+d_{+}-d_{-}}\left[\bar{D}_{12}^{(2)}+\left(\bar{M}^{(1)} \bar{D}^{(1)}\right)_{12}\right] \\
\frac{1}{2+d_{-}-d_{+}}\left[\bar{D}_{21}^{(2)}+\left(\bar{M}^{(1)} \bar{D}^{(1)}\right)_{21}\right] & \frac{1}{2}\left[\bar{D}_{22}^{(2)}+\left(\bar{M}^{(1)} \bar{D}^{(1)}\right)_{22}\right]
\end{array}\right) .
$$

The evolution equation may then be written as, to NNLO,

$$
\begin{aligned}
\mu(n, a) & =C(n, a) S(n) \bar{M}(n, a)^{-1}\left(a_{0} / a\right)^{\hat{D}^{(0)}(n)} \bar{M}\left(n, a_{0}\right) S^{-1}(n) C\left(n, a_{0}\right)^{-1} \mu\left(n, a_{0}\right), \\
a & =\alpha_{s}\left(Q^{2}\right) / 4 \pi, \quad a_{0}=\alpha_{s}\left(Q_{0}^{2}\right) / 4 \pi
\end{aligned}
$$

and $\alpha_{s}$ is to be calculated to three loops:

$$
\alpha_{s}\left(Q^{2}\right)=\frac{4 \pi}{\beta_{0} L}\left\{1-\frac{\beta_{1} \log L}{\beta_{0}^{2} L}+\frac{\beta_{1}^{2} \log ^{2} L-\beta_{1}^{2} \log L+\beta_{2} \beta_{0}-\beta_{1}^{2}}{\beta_{0}^{4} L^{2}}\right\}
$$

with

$$
\begin{aligned}
L & =\log \frac{Q^{2}}{\Lambda^{2}} ; \quad \beta_{0}=11-\frac{2}{3} n_{f}, \\
\beta_{1} & =102-\frac{38}{3} n_{f}, \quad \beta_{2}=\frac{2857}{2}-\frac{5033}{18} n_{f}+\frac{325}{54} n_{f}^{2} .
\end{aligned}
$$

We work in the $\overline{\mathrm{MS}}$ scheme throughout this paper. For the NS component the corresponding equation is

$$
\begin{aligned}
\mu_{N S}\left(n, Q^{2}\right) & =\left(\frac{\alpha_{s}\left(Q_{0}^{2}\right)}{\alpha_{s}\left(Q^{2}\right)}\right)^{-\gamma_{N S}^{(0)}(n) / 2 \beta_{0}} \\
& \times \frac{1+B_{n}^{(1)} \alpha_{s}\left(Q^{2}\right) / 4 \pi+B_{n}^{(2)}\left(\alpha_{s}\left(Q^{2}\right) / 4 \pi\right)^{2}}{1+B_{n}^{(1)} \alpha_{s}\left(Q_{0}^{2}\right) / 4 \pi+B_{n}^{(2)}\left(\alpha_{s}\left(Q_{0}^{2}\right) / 4 \pi\right)^{2}} \mu_{N S}\left(n, Q_{0}^{2}\right) .
\end{aligned}
$$

The explicit values of the $B_{n}^{(i)}$, expressible in terms of the $\gamma_{N S}^{(i)}, C_{N S}^{(i)}(n)$, may be found in ref. 10. We can rewrite $(2.12 \mathrm{a})$ expanding the denominator as

$$
\begin{aligned}
\mu_{N S}\left(n, Q^{2}\right) & =\left(\frac{\alpha_{s}\left(Q_{0}^{2}\right)}{\alpha_{s}\left(Q^{2}\right)}\right)^{-\gamma_{N S}^{(0)}(n) / 2 \beta_{0}} \\
& \times\left\{1+B_{n}^{(1)} \alpha_{s}\left(Q^{2}\right) / 4 \pi-B_{n}^{(1)} \alpha_{s}\left(Q_{0}^{2}\right) / 4 \pi\right. \\
& +B_{n}^{(2)}\left(\alpha_{s}\left(Q^{2}\right) / 4 \pi\right)^{2}-B_{n}^{(2)}\left(\alpha_{s}\left(Q_{0}^{2}\right) / 4 \pi\right)^{2}+\left(B_{n}^{(1)} \alpha_{s}\left(Q_{0}^{2}\right) / 4 \pi\right)^{2} \\
& \left.\left.-\left(B_{n}^{(1)} \alpha_{s}\left(Q_{0}^{2}\right) / 4 \pi\right)\left(B_{n}^{(1)} \alpha_{s}\left(Q^{2}\right) / 4 \pi\right)\right)\right\} \mu_{N S}\left(n, Q_{0}^{2}\right) .
\end{aligned}
$$

The difference between $(2.12 \mathrm{~b})$ and $(2.12 \mathrm{a})$ is of order $\alpha_{s}^{3}$, and may be used to estimate the effect of higher order (NNNLO) corrections.

A last preliminary point to be discussed is that of the number of flavours. We will be working with the moments $\mu\left(n, Q^{2}\right)$, so we have only one momentum variable. We then split the $Q^{2}$ range into the following two intervals:

$$
Q^{2} \lesssim m_{b}^{2}(\mathrm{I}) ; \quad m_{b}^{2} \lesssim Q^{2}(\mathrm{II})
$$

The values of $Q^{2}$ we will be using will be much less than $m_{t}^{2}$, and larger than or of the order of $m_{c}^{2}$ so we need not consider $c, t$ quark thresholds. Then, in region (I) we take $n_{f}=4$ and in region (II), $n_{f}=5$. The matching will be carried over following the prescription of ref. 13: to NNLO,

$$
\beta_{0}^{n_{f}+1} \log \frac{\Lambda^{2}\left(n_{f}+1\right)}{\Lambda^{2}\left(n_{f}\right)}=\left(\beta_{0}^{n_{f}+1}-\beta_{0}^{n_{f}}\right) L_{h}+\delta_{N L O}+\delta_{N N L O}
$$

where

$$
\begin{aligned}
\delta_{N L O} & =\left(b_{1}^{n_{f}+1}-b_{1}^{n_{f}}\right) \log L_{h}-b_{1}^{n_{f}+1} \log \frac{\beta_{0}^{n_{f}+1}}{\beta_{0}^{n_{f}}} \\
\delta_{N N L O} & =\frac{1}{\beta_{0}^{n_{f}} L_{h}}\left[\left(b_{1}^{n_{f}+1}-b_{1}^{n_{f}}\right) b_{1}^{n_{f}} \log L_{h}+\left(b_{1}^{n_{f}+1}\right)^{2}-\left(b_{1}^{n_{f}}\right)^{2}+b_{2}^{n_{f}}-b_{2}^{n_{f}+1}+\frac{7}{24}\right] .
\end{aligned}
$$


Here,

$$
L_{h}=\log \left[m^{2}\left(n_{f}+1\right) / \Lambda^{2}\left(n_{f}\right)\right], \quad b_{i}=\beta_{i} / \beta_{0}
$$

and $m\left(n_{f}+1\right)$ is the pole mass of the $\left(n_{f}+1\right)$ th quark.

\section{Experimental input}

\subsection{The method of Bernstein polynomials}

Because, for a given value of $Q^{2}$, only a limited number of experimental points, covering a partial range of values of $x$, are available, one cannot simply use the moments equations. A method devised to deal with a situation like the present one is that of averages with the (modified) Bernstein polynomials. ${ }^{3}$ We define the (Bernstein) polynomials as

$$
\begin{aligned}
p_{n k}(x) & =\frac{2 \Gamma\left(n+\frac{3}{2}\right)}{\Gamma\left(k+\frac{1}{2}\right) \Gamma(n-k+1)} x^{2 k}\left(1-x^{2}\right)^{n-k} \\
& =\frac{2(n-k) ! \Gamma\left(n+\frac{3}{2}\right)}{\Gamma\left(k+\frac{1}{2}\right) \Gamma(n-k+1)} \sum_{l=0}^{n-k} \frac{(-1)^{l}}{l !(n-k-l) !} x^{2(k+l)} ; \quad k \leq n .
\end{aligned}
$$

These polynomials have a number of useful properties. First, they are positive and have a single maximum located at

$$
\bar{x}_{n k}=\frac{\Gamma(k+1) \Gamma\left(n+\frac{3}{2}\right)}{\Gamma\left(k+\frac{1}{2}\right) \Gamma(n+2)} ;
$$

they are concentrated around this point, with a spread of

$$
\Delta x_{n k}=\sqrt{\frac{k+\frac{1}{2}}{n+\frac{3}{2}}-\left[\frac{\Gamma(k+1) \Gamma\left(n+\frac{3}{2}\right)}{\Gamma\left(k+\frac{1}{2}\right) \Gamma(n+2)}\right]^{2}},
$$

and they are normalized to unity, $\int_{0}^{1} \mathrm{~d} x p_{n k}(x)=1$. Therefore, the integral

$$
\int_{0}^{1} \mathrm{~d} x p_{n k}(x) \varphi(x)
$$

represents an average of the function $\varphi(x)$ in the region $\bar{x}_{n k}-\frac{1}{2} \Delta x_{n k} \lesssim x \lesssim \bar{x}_{n k}+\frac{1}{2} \Delta x_{n k}$; the values of the function $\varphi(x)$ outside this interval contribute little to the integral, as $p_{n k}(x)$ decreases to zero very quickly there. Finally, and using the binomial expansion in Eq. (3.1), it follows that the averages with the $p_{n k}$ of a function can be obtained in terms of its even moments:

$$
\begin{aligned}
\int_{0}^{1} \mathrm{~d} x p_{n k}(x) \varphi(x) & =\frac{2(n-k) ! \Gamma\left(n+\frac{3}{2}\right)}{\Gamma\left(k+\frac{1}{2}\right) \Gamma(n-k+1)} \sum_{l=0}^{n-k} \frac{(-1)^{l}}{l !(n-k-l) !} \varphi_{2 k+2 l}, \\
\varphi_{2 l} & =\int_{0}^{1} \mathrm{~d} x x^{2 l} \varphi(x) .
\end{aligned}
$$

We will thus consider our experimental input to be given by averages

$$
F_{n k}^{(\exp )}\left(Q^{2}\right) \equiv \int_{0}^{1} \mathrm{~d} x p_{n k}(x) F_{2}^{(\exp )}\left(x, Q^{2}\right),
$$

with $F_{2}^{(\exp )}\left(x, Q^{2}\right)$ the experimental structure function.

\footnotetext{
${ }^{3}$ We call the polynomials modified because, since only even moments are known, we have to consider polynomials in the variable $x^{2}$. For more details on the method, see refs. 11, 14 .
} 


\section{-J. SANTIAGO AND F. J. YNDURÁIN-}

\subsection{Calculation of the experimental averages}

Even with the method of Bernstein polynomials, the calculation of an average such as (3.2) requires some interpolation and extrapolation of $F_{2}^{(\exp )}\left(x, Q^{2}\right)$. To do so we have used two different methods. In both we separate $F_{2}$ into a singlet and a nonsinglet part, writing

$$
F_{2}=F_{S}+F_{N S}
$$

In the first method we use, for each value of $Q^{2}$ independently, a phenomenological expression for the $F$,

$$
\begin{aligned}
& F_{S}^{\text {phen. }}(x)=\left(A x^{-0.44}+C\right)(1-x)^{\nu}, \\
& F_{N S}^{\text {phen. }}(x)=B x^{0.5}(1-x)^{\mu} .
\end{aligned}
$$

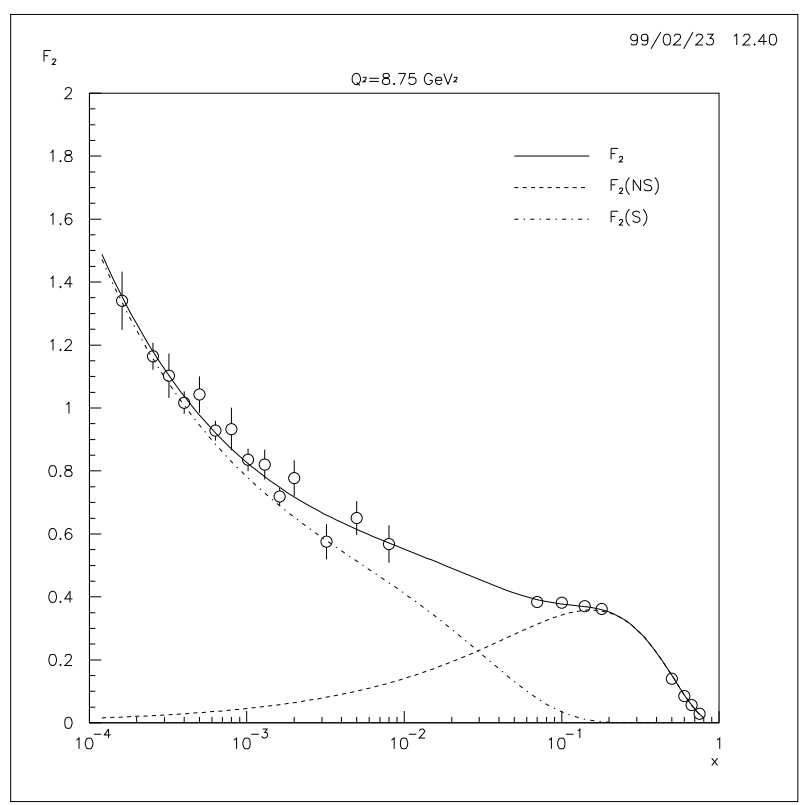

Figure 1 Phenomenological fit for $Q^{2}=8.75 \mathrm{GeV}$ (logarithmic scale).

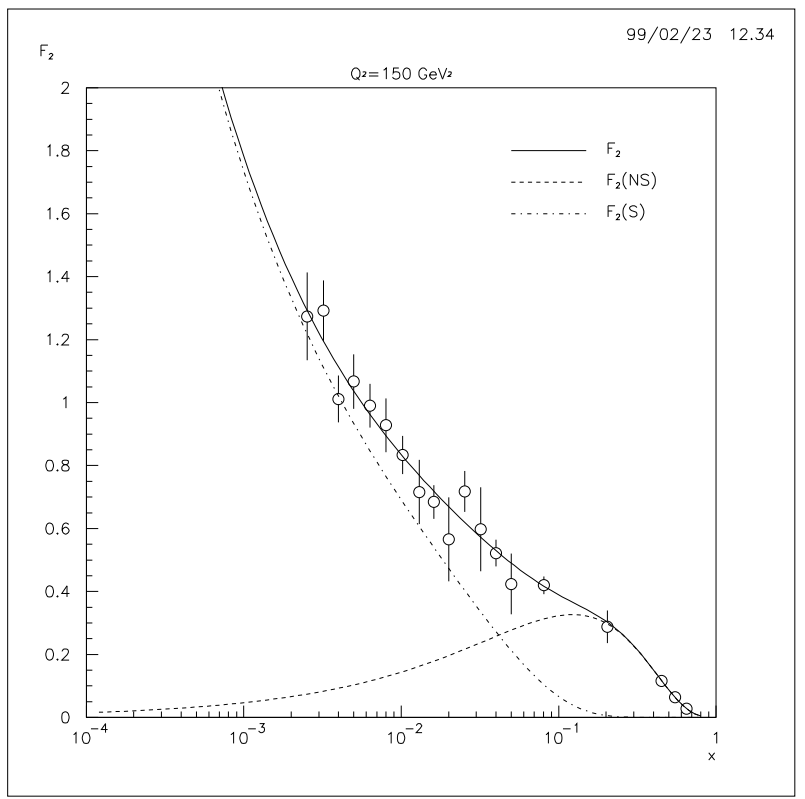

Figure 2 Phenomenological fit for $Q^{2}=150 \mathrm{GeV}$ (logarithmic scale). 
A theoretical justification of (3.3) may be found in ref. 7 and work quoted there; but we want to emphasize that, in the present paper, (3.3) is to be considered as only a convenient interpolation of the data to allow calculation of the integrals (3.2). In fact, the parameters $A, B, C, \nu, \mu$ are taken to be totally free, and assumed to be uncorrelated for different values of $Q^{2}$. Thus, no theoretical bias is induced in the $Q^{2}$ dependence. The fits, for a couple of representative $Q^{2}$ values, are given in Figs. 1, 2. In Fig. 3 we also show the polynomial $p_{31}(x)$, superimposed on the fit and the experimental values of $F_{2}\left(x, Q^{2}=8.75 \mathrm{GeV}^{2}\right)$, as a representative case.

The second method we consider is to use the parameterization of data (that we denote by MRST98) given in ref. 15, which includes some theoretical input. Then the integral involved in the Bernstein average is evaluated with the help of the parametric expression, for each value of $Q^{2}$.

We consider the first method to be the cleanest one; the results found using the MRST98 parameterization are presented mostly to show the insensitivity of the evaluation to the method of obtaining the "experimental" averages $F_{n k}^{(\exp )}\left(Q^{2}\right)$.

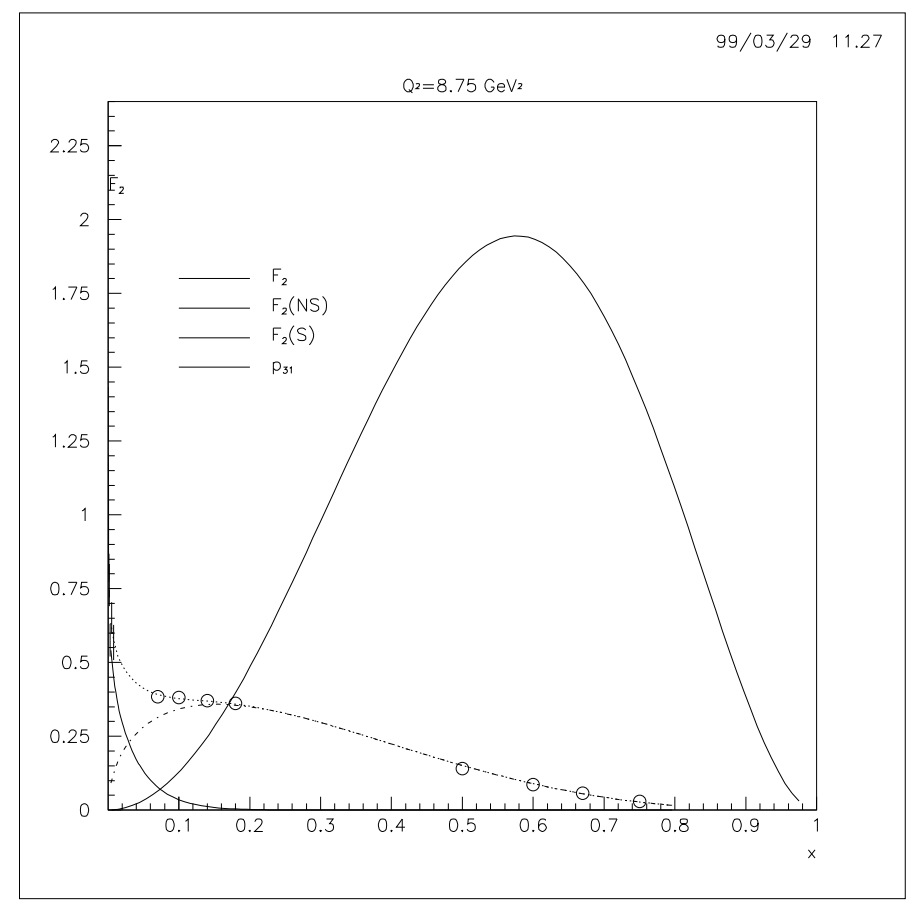

Figure 3 Phenomenological fit for $Q^{2}=8.75 \mathrm{GeV}$, and Bernstein polynomial $p_{31}$.

Only this polynomial is shown as an example, although for this value of $Q_{0}^{2}$ other Bernstein averages are also meaningful. The points at very small $x$ are not shown (compare with Fig. 2).

\subsection{Experimental input}

To begin with, we have the limitation that, since the $\gamma \mathrm{s}$ in the QCD evolution equations are only known for $n=2,4,6,8$, we will be only able to use, for the theoretical calculations, the ten Bernstein polynomials ${ }^{4}$

$$
\begin{aligned}
& p_{n k}(x) ; \quad k=0, n=0,1,2,3 ; \\
& k=1, n=1,2,3 ; \\
& k=2, \quad n=2,3 ; \\
& k=3, \quad n=3 .
\end{aligned}
$$

\footnotetext{
${ }^{4}$ Actually, $\gamma_{N S}^{(2)}(n)$ is also known for $n=10$ but we will not be able to use this because in $F_{2}$ for electroproduction
} singlet and nonsinglet are not separated. 
However, we will not have experimental information, for a given $Q^{2}$, on all of the corresponding averages. Thus, for example, at very large values of $Q^{2}$, the HERA measurements have been carried over only for small values of $x$, so here only the averages with $p_{30}$ and at most $p_{20}$ can be used. Also, there are generally speaking no data for $x$ near unity, so averages with large $n-k$ cannot be used either. As a matter of fact, we have only used the six polynomials

$$
\begin{aligned}
& p_{10}, p_{20}, p_{30} ; \\
& p_{21}, p_{31}, p_{32} .
\end{aligned}
$$

When we take this into account, it follows that we can calculate reliably only a total of 102 Bernstein averages, using the experimental data from SLAC ${ }^{[16]}, \mathrm{BCDMS}^{[17]}$ E665 ${ }^{[18]}$ and HERA ${ }^{[19]}$. The experimental averages, as defined in Eq. (3.2) with the interpolation given by (3.3), are shown in Fig. 4 (together with the NNLO fit, see below); the errors there are the errors in the integrals (3.2) induced by the errors in the parameters $A, B, C, \nu, \mu$; and these errors are obtained, in turn, from the experimental errors of the fitted $F_{2}$. These errors of $F_{n k}^{(\exp )}$ are what we consider the statistical, experimental errors of our input. There is however one more point that has to be discussed in this respect. We have, for each $Q^{2}$, theoretical information on four moments, but we use six Bernstein averages: so we are double counting part of our information. This is repaired as follows: we consider the values of $Q^{2}$ for which we can use more than four Bernstein averages, and consider the number above four of these to be "duplicate" information. The total number of these duplicates is of 34 . Then we renormalize the experimental error by multiplying it by $\sqrt{102 /(102-34)}=1.22$.

\section{Theoretical calculation. Error analysis}

The QCD equations $(2.11,12)$ only give the evolution of the moments. Therefore, we have to take their initial values, at a fixed $Q_{0}^{2}$

$$
\mu_{S}\left(n, Q_{0}^{2}\right), \quad \mu_{G}\left(n, Q_{0}^{2}\right) ; \quad \mu_{N S}\left(n, Q_{0}^{2}\right) ; \quad n=2,4,6,8,
$$

as parameters. For the actual calculations we chose $Q_{0}^{2}=8.75 \mathrm{GeV}^{2}$ because, for this value, there are experimental data in a wide range of values of $x$ so the input values are directly constrained by experiment. We have considered varying the value of $Q_{0}^{2}$; this provides an indication of the errors induced by the approximations made for, in an exact calculation, the result should be independent of $Q_{0}^{2}$. Indeed, the variation is small; for example, if we take $Q_{0}^{2}=12 \mathrm{GeV}^{2}$ the QCD mass $\Lambda$ varies by $11 \mathrm{MeV}$.

Of these twelve moments only eleven are free. In fact, because of the momentum sum rule it follows that

$$
\lim _{Q^{2} \rightarrow \infty}\left[\mu_{S}\left(2, Q^{2}\right)+\mu_{G}\left(2, Q^{2}\right)\right]=\left\langle Q_{q}^{2}\right\rangle=\frac{1}{n_{f}} \sum_{q=1}^{n_{f}} Q_{q}^{2},
$$

where $\left\langle Q_{q}^{2}\right\rangle$ is the average quark charge; for $n_{f}=4,\left\langle Q_{q}^{2}\right\rangle=\frac{5}{18}$; for $n_{f}=5,\left\langle Q_{q}^{2}\right\rangle=\frac{11}{45}$. To implement this, we may use the equations (2.11) with $\alpha_{s}\left(Q^{2}\right) \rightarrow 0$, and that $d_{+}(2)=0, d_{-}(2)<0$ together with the values of the $S_{i j}(2)$ to get the constraint

$$
\frac{5}{18}=\frac{16+3 n_{f}}{3 n_{f}} \sum_{j=S, G}\left(\bar{M}\left(\left(2, a_{0}\right) S^{-1}(2) C^{-1}\left(2, a_{0}\right)\right)_{1 j} \mu_{j}\left(2, Q_{0}^{2}\right)\right.
$$

and the quantities here are to be calculated for $n_{f}=4$. So we may use (4.1) to eliminate $\mu_{G}\left(2, Q_{0}^{2}\right)$ in favour of $\mu_{S}\left(2, Q_{0}^{2}\right)$, leaving 11 free moments, as stated. To these we have to add a further parameter viz., the QCD mass $\Lambda$, to a total of 12 free parameters for the fit.

The procedure is now rather straightforward. We calculate the $\mu\left(n, Q^{2}\right)$ in terms of the $\mu\left(n, Q_{0}^{2}\right)$ using $(2.11,12)$; and once these $\mu\left(n, Q^{2}\right)$ known, we evaluate the theoretical Bernstein moments $F_{k n}^{\text {th }}\left(Q^{2}\right)$ for all $Q^{2}$, in terms of our twelve parameters. We are then ready to fit the experimental quantities obtained before, $F_{n k}^{(\exp )}\left(Q^{2}\right)$; a fit which is performed with the MINUIT program ${ }^{[20]}$. Before presenting the results, however, we have to discuss a number of theoretical questions, including refinements and estimates of theoretical errors. 
-CALCUlATION OF ELECTROPRODUCTION TO NNLO AND PRECISION DETERMINATION OF $\alpha_{s}{ }^{-}$

\subsection{Target mass corrections}

Target mass corrections (TMC) are of order $n m_{p} / Q^{2}$, for the $n$th moment $\left(m_{p}\right.$ being the mass of the proton) and must be included in a precision calculation. The corrections can be evaluated exactly ${ }^{[21]}$, to LO in $\alpha_{s}$, but we here will only take them into account to first order in $m_{p}^{2} / Q^{2}$. The reasons are that higher twist (HT) corrections, of order $n \Lambda^{2} / Q^{2}$, are not known; and neither are the NLO (in $\alpha_{s}$ ) TMCs. Although it may be argued that HT are suppressed with respect to TMC by $1 / N_{c}$, with $N_{c}=3$ the number of colours in the large $N_{c}$ limit, it is useless to include effects $O\left(m_{p}^{4} / Q^{4}\right)$ while the ones of order $\Lambda^{2} / Q^{2}$ and $O\left(\alpha_{s} m_{p}^{2} / Q^{2}\right)$ corrections are not known. Anyway, we will give results both with and without TMC; the variation is slight, and the error, as just estimated, negligible.

We write (see refs. 12, 21)

$$
\mu_{N S}^{T M C}\left(n, Q^{2}\right)=\mu_{N S}\left(n, Q^{2}\right)+\frac{n(n-1)}{n+2} \frac{m_{p}}{Q^{2}} \mu_{N S}\left(n+2, Q^{2}\right) .
$$

It should be noted that the TMCs for the singlet are negligible compared to those of the NS, which is why we only take into account the last. Another point is that (4.2) involves one further moment, $\mu_{N S}\left(10, Q^{2}\right)$. Since we do not want to add a new parameter to the fit, what we do is to estimate $\mu_{N S}\left(10, Q^{2}\right)$ from the fitted expression for $F_{2}$, Eq. (3.3). Since this is only a correction, it does not matter much if it is not very accurate.

\subsection{Correlation between gluon structure function and $\Lambda$}

As is known, in other fits there is a strong correlation between the value of the QCD parameter $\Lambda$ and the gluon structure function, which leads to instabilities in the values of the last; this is due to the fact that the $\mu_{G}$ are not directly related to a measured quantity. The problem is however not serious in our case. This is because, in our evaluations, $x G\left(x, Q^{2}\right)$ is only represented by its three input moments

$$
\mu_{G}\left(4, Q_{0}\right), \quad \mu_{G}\left(6, Q_{0}\right), \quad \mu_{G}\left(8, Q_{0}\right) ;
$$

recall that $\mu_{G}\left(2, Q_{0}\right)$ can be eliminated in favour of $\mu_{S}\left(2, Q_{0}\right)$ using the momentum sum rule. We have also found that the instabilities disappear completely if we simply make the requirement that $\mu_{G}\left(n, Q^{2}\right)>$ $\mu_{G}\left(n+2, Q^{2}\right)$, a requirement that follows from the positivity of $G\left(x, Q^{2}\right)$ as a probability density for gluons with momentum fraction $x$. In fact, from the positivity of $G$ we can impose other inequalities; ${ }^{[14]}$ for example, considering only linear ones, we must have

$$
\int_{0}^{1} d x p_{n k}(x) F_{G}\left(x, Q_{0}^{2}\right)=\frac{2(n-k) ! \Gamma\left(n+\frac{3}{2}\right)}{\Gamma\left(k+\frac{1}{2}\right) \Gamma(n-k+1)} \sum_{l=0}^{n-k} \frac{(-1)^{l}}{l !(n-k-l) !} \mu_{G}\left(2 k+2 l+2, Q_{0}^{2}\right) \geq 0 .
$$

We have checked that his is verified by the central values for the $\mu_{G}$ in our fits.

If we allowed the $\mu_{G}$ to vary freely this would result in the appearance of a spurious minimum for which the lowest moment would be smaller than the higher ones. Nevertheless, even in this case the value of $\Lambda$ does not vary too much: it only decreases by some $10 \mathrm{MeV}$. In our fits we of course require positivity.

\subsection{Quark mass dependence}

Since our $Q^{2}$ range goes through the $b$ quark mass threshold, we have to worry about that region. In our main calculation we have simply used the matching conditions (2.13), fixing the mass of the $b$ quark from the recent determination in ref. 22 , correct to $O\left(\alpha_{s}^{4}\right)$,

$$
m_{b}=5001_{-66}^{+101} \mathrm{MeV} \text {. }
$$

It is to be noted, however, that the use of (2.13) is not enough to take into account exactly the mass dependence. To do so, one would have to rewrite (and solve) the renormalization group equations taking into account the finiteness of $m_{b}$, a very hard task quite outside our scope here. The fact that the use of (2.13) gives only an approximation to the mass dependence results in a discontinuity of the theoretical calculation, at NNLO, near $Q^{2}=m_{b}^{2}$, which can be seen clearly in Fig. 4 . Since the discontinuity is of $\lesssim 1 \%$, and affects only the fit to very few experimental points, we consider the use of (2.13) to be the best 
available procedure. Alternatively, we could simply avoid the region around the $b$ quark threshold and thus cut off the interval ${ }^{5}$

$$
m_{b}^{2} \leq Q^{2} \leq 4 m_{b}^{2}
$$

Actually, we remove from the fit the values of the moments for $Q^{2}$ between 20 and $90 \mathrm{GeV}^{2}$. Again, we take the difference between the results with both methods (matching or cutting the threshold) as part of the measure of the error due to the $b$ quark mass effect. The rest of the error is obtained varying $m_{b}$ inside its error bars, as above.

\subsection{Higher order corrections}

Higher order corrections are of two types. First, we have higher order in $\Lambda^{2} / Q^{2}$, viz., higher twist effects (HT). Since so little is known about these we simply take into account phenomenologically the more important ones by adding, to $\mu_{N S}\left(n, Q^{2}\right)$, the correction

$$
\mu_{N S}^{H T}\left(n, Q^{2}\right)=n\left(a \Lambda^{2} / Q^{2}\right) \mu_{N S}\left(n, Q^{2}\right)
$$

with $a$ an unknown parameter, to be fitted, and expected to be of order unity; in our fit we find $a \sim-0.20$, see below. The difference between the results with this new contribution, and the one without, will be the estimated error induced by HT effects. Note that our central values (Table 1 below) are obtained without including the HT term. This is because, although the value we get for $a$ is reasonable, the fact remains that the expression (4.3) used for HT is little more than educated guesswork, and we want to avoid as much as possible to introduce biases in our calculation of $\Lambda, \alpha_{s}$.

The second type of higher order corrections will be corrections of relative order $\alpha_{s}^{3}$, i.e., NNNLO corrections. We estimate these by the different results obtained performing our calculations either with the fractional expression $(2.12 \mathrm{~b})$, or with the expanded denominator one, $(2.12 \mathrm{c})$. This simulates, at least in the expected behaviour at large $n$, the largest NNNLO corrections; in fact, the largest corrections of order $j$ to $F_{2}$ may be argued to behave as $\alpha_{s}^{j} \log ^{j}(1-x)$ and stem from the $C_{N S}^{(j)}(n), \gamma_{N S}^{(j)}(n)$ at large $n$; see for example ref. 12, Sect. 4.9ii and references therein. By using our procedure, we are estimating e.g.,

$$
C_{N S}^{(3)}(n) \sim C_{N S}^{(2)}(n) C_{N S}^{(1)}(n) .
$$

\subsection{Dependence on parameterizations}

The dependence of our input data for the Bernstein averages on the parameterizations used to evaluate the corresponding integrals is estimated as follows: we calculate using our interpolation formulas, Eq. (3.3), and then repeat the calculation with the MRST98 parameterization. The difference (10 MeV for $\Lambda$ ) will be our estimated error due to this source.

${ }^{5}$ We could also consider increasing the minimum value of $Q^{2}$ to be well clear of the $c$ quark threshold, but we have not bothered to do so as the experimental points in the corresponding region are not significant; cf. Fig. 4. 
-CALCULATION OF ELECTROPRODUCTION TO NNLO AND PRECISION DETERMINATION OF $\alpha_{s}{ }^{-}$

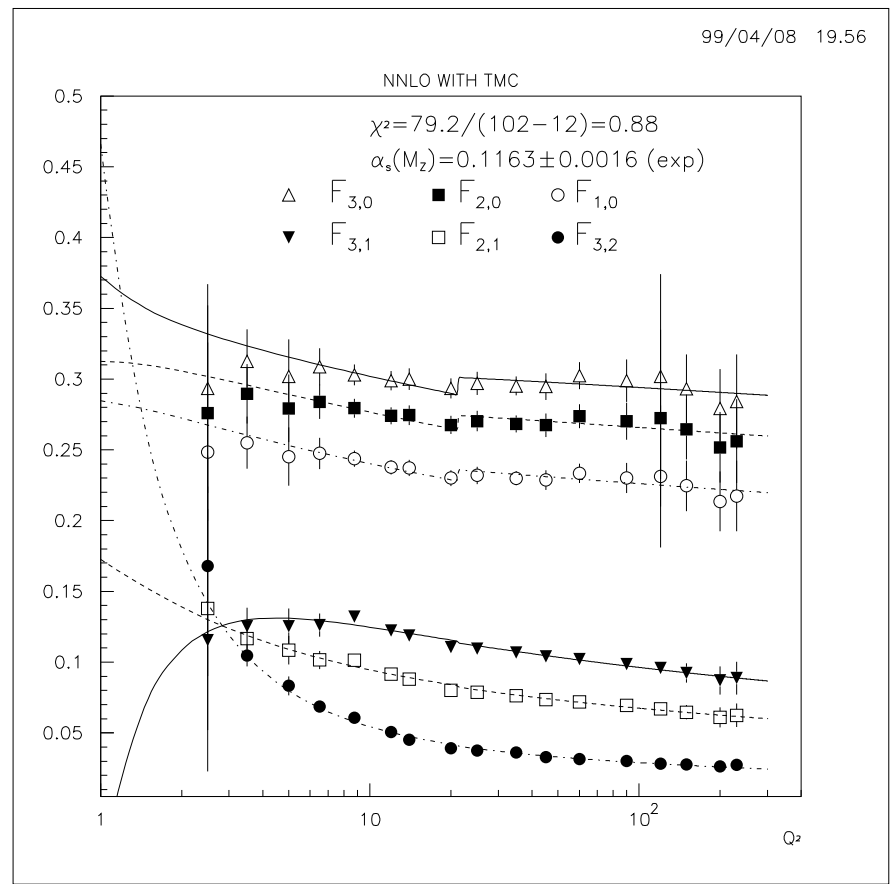

FIGURE 4 Experimental values for the Bernstein averages, and NNLO theoretical curves, with the gluon structure function constrained and TMC taken into account.

\section{Results. Discussion}

We present in Table 1 a compilation of the results obtained with our calculations at LO, NLO and NNLO, with TMCs taken into account; the fit to the data itself is shown, for the NNLO calculation (with TMC) in Fig. 4. In this Table 1, only statistical errors are shown; systematic (theoretical) errors will be discussed below. We note that the errors given in Table 1 are "renormalized" to take into account the effective number of independent experimental points, as discussed at the end of Sect. 3. That is to say, the error presented is obtained from the raw error by multiplying it by 1.22 . In calculating the $\chi^{2} /$ d.o.f., however, we have considered all the input experimental points, as both the total chi-squared and the number of degrees of freedom change by the same amount.

\begin{tabular}{|c|c|l|l|}
\hline Order & $\Lambda\left(n_{f}=4\right)$ & $\alpha_{s}\left(M_{Z}^{2}\right)$ & $\chi^{2} /$ d.o.f. \\
\hline LO & $215 \pm 73$ & $0.130 \pm 0.005$ & $212 /(102-12)$ \\
\hline NLO & $282 \pm 40$ & $0.116 \pm 0.0034$ & $80.0 /(102-12)$ \\
\hline NNLO & $283 \pm 25$ & $0.1172 \pm 0.0017$ & $79.2 /(102-12)$ \\
\hline
\end{tabular}

Table 1

The NLO corrections are very clearly seen in the fit: the $\chi^{2} /$ d.o.f. decreases from a largish value of $\sim 2.4$ to a very good $\sim 0.89$ when including these. The fit is so good already at this order that there is very little room for improvement when going to NNLO; nevertheless, an improvement is seen. Not in the $\chi^{2} /$ d.o.f., which only decreases minutely (to $\chi^{2} /$ d.o.f. $\sim 0.88$ ) but certainly in that including NNLO corrections leads to a noticeable gain both in the quality of the determination of the coupling, and in the stability of the fits.

The estimated systematic errors, originating from various sources, are shown for the NNLO case in Table 2: 
-J. SANTIAGO AND F. J. YNDURÁIN-

\begin{tabular}{|c|c|c|c|}
\hline Source of error & $\Lambda\left(n_{f}=4 ; 3\right.$ loop $)$ & $\Delta \Lambda\left(n_{f}=4 ; 3\right.$ loop $)$ & $\Delta \alpha_{s}\left(M_{Z}^{2}\right)$ \\
\hline No TMC & 292 & 9 & 0.0006 \\
\hline Interpol. (MRST98) & 273 & 10 & 0.0007 \\
\hline HT & 292 & 9 & 0.0006 \\
\hline Quark mass effect & 299 & 16 & 0.0011 \\
\hline$Q_{0}^{2}$ to $12 \mathrm{GeV}^{2}$ & 294 & 11 & 0.0008 \\
\hline$N \mathrm{NNLO}$ & 289 & 6 & 0.0004 \\
\hline
\end{tabular}

Table 2

Let us comment on the meaning of the different entries. No TMC means that we have not taken target mass corrections into account. The corresponding error is not included when evaluating the overall theoretical error because, since we take into account TMC in our central value, the error would be of order $T M C^{2}$, or $\alpha_{s} \times T M C$, quite negligible. MRST98 means that we have used the MRST98 interpolation (Subsect. 3.2) to calculate the integrals (3.2). HT means that we have taken into account the presence of higher twist by adding a contribution like (4.2). The fitted value of the phenomenological parameter $a$ is $a=-0.202 \pm 0.030 .{ }^{6}$ "Quark mass effect" means that we have cut off the $b$ quark threshold as discussed in Subsect. 4.3; the error in Table 2 takes into account also the variations due to the error in the $m_{b}$ mass. $Q_{0}^{2}$ to $12 \mathrm{GeV}^{2}$ means that we take the input moments defined at this value of the momentum, $\mu_{i}\left(n, Q_{0}^{2}=12 \mathrm{GeV}^{2}\right), i=S, G, N S$. Finally, NNNLO means that we have fitted with the expanded formula (2.12c) instead of the fractional one (2.12b). Note that we only take this into account for the nonsinglet. This is because for large $n$, which is when the higher order corrections are larger, the NS piece of the structure function is the dominating one.

Composing quadratically systematic (theoretical) and statistical (experimental) errors we find the best result for the QCD coupling,

$$
\begin{aligned}
\Lambda\left(n_{f}=4,3 \text { loop }\right) & =282.7 \pm 25.1 \text { (stat.) } \pm 24.5 \text { (syst.) }=282.7 \pm 35.1 \mathrm{MeV} ; \\
\alpha_{s}^{(3 \text { loop })}\left(M_{Z}\right) & =0.1172 \pm 0.0017 \text { (stat.) } \pm 0.0017 \text { (syst.) }=0.1172 \pm 0.0024 ;
\end{aligned}
$$

the corresponding central value for $\Lambda\left(n_{f}=5,3 \mathrm{loop}\right)$ is of $0.200 \mathrm{MeV}$. It is to be noted that composing the "theoretical" errors as if they were independent leads to a certain amount of double-counting. Thus, the results should be independent of the value of $Q_{0}^{2}$ if the calculation was to all orders, so the two last errors in Table 2 are connected. We have, however, preferred to play it safe, particularly because these errors are in some cases little more than rough estimates.

We next show a table comparing our results to previous determinations for $\alpha_{s}\left(M_{Z}^{2}\right)$; these are taken from the review by Bethke ${ }^{[23]}$, including only the processes where the theoretical calculation has been pushed to the NNLO level and the experimental data are good enough to make the analysis meaningful. ${ }^{7}$ We also incorporate in the table the recent results in ref. 10:

${ }^{6}$ This is a very reasonable value that makes the mass associated with higher twists, $M_{H T}=\Lambda \sqrt{|a|} \sim 0.13 \mathrm{GeV}$ of the order of typical HT parameters: intrinsic average transverse momentum of the quarks, or inverse proton radius; see e.g. the paper of De Rújula et al., ref. 1.

7 This excludes $e^{+} e^{-}$annihilations. 
-CALCULATION OF ELECTROPRODUCTION TO NNLO AND PRECISION DETERMINATION OF $\alpha_{s^{-}}$

\begin{tabular}{|c|c|c|c|c|}
\hline Process & \multicolumn{3}{|c|}{$\begin{array}{c}\text { Average } Q^{2} \\
\text { or } Q^{2} \text { range }[\mathrm{GeV}]^{2}\end{array}$} & $\alpha_{s}\left(M_{Z}^{2}\right)$ \\
\hline $\mathrm{DIS} ; \nu, \mathrm{Bj}$ & \multicolumn{3}{|c|}{2.5} & 0.122 \\
\hline DIS; $\nu$, GLS & \multicolumn{3}{|l|}{3} & 0.115 \\
\hline$\tau$ decays & \multicolumn{3}{|c|}{$(1.777)^{2}$} & 0.119 \\
\hline$Z \rightarrow$ hadrons & \multicolumn{3}{|c|}{$(91.2)^{2}$} & 0.124 \\
\hline $\mathrm{DIS} ; \nu, x F_{3}$ & 5 & & 100 & $0.117 \pm 0.010$ \\
\hline our result & 2.5 & - & 230 & $0.1172 \pm 0.0024$ \\
\hline
\end{tabular}

Table 3

Here DIS means deep inelastic scattering, Bj stands for the Bjorken, and GLS for the Gross-Llewellyn Smith sum rules. The $x F_{3}$ result is that of ref. 10 .

The previously existing average value, also taking into account NLO calculations, was

$$
\alpha_{s}\left(M_{Z}^{2}\right)=0.118 \pm 0.006
$$

when including both our result and that of ref. 10 the new average and error become

$$
\alpha_{s}\left(M_{Z}^{2}\right)=0.1174 \pm 0.0016 \text {. }
$$

We add a further comment. If we only kept, in the old determinations of $\alpha_{s}$, processes with spacelike momenta then, as noted by Bethke, ${ }^{[23]}$ a slightly smaller value was obtained for the coupling:

$$
\alpha_{s}\left(M_{Z}^{2}\right)=0.114 \pm 0.005 \text { (spacelike momenta). }
$$

Our results correct this to some extent, by increasing slightly the average value for "spacelike" determinations.

To finish this paper, we discuss briefly why our calculation yields such accurate results. First of all, and compared with neutrino data, our experimental input turns out to be much more precise, and also it extends over a much wider range of $Q^{2}$ values. Actually, this wide range of values is one of the assets in our evaluation, particularly when comparing it to $Z, \tau$ decays where only one value of $Q^{2}$ is essentially available. Our range is such that not only leading logs, but next-to-leading logs vary appreciably: cf Table 1 . The inclusion of NNL logs then stabilizes the results and decreases the errors and (very slightly) the $\chi^{2} /$ d.o.f.. 
-J. SANTIAGO AND F. J. YNDURÁIN-

\section{Acknowledgments}

The authors are indebted to A. Vermaseren for several illuminating discussions, and to L. Labarga for the same reason and for informatic help. Prof. Blümlein has also contributed with useful remarks about target mass corrections. Finally, thanks are also due to S. Bethke who spotted (and allowed us to correct) a mistake in the paper.

The financial support of CICYT (Spain, contracts AEN 97-1678 and AEN 96-1672) is gratefully acknowledged. One of us (J. S.) wishes also to thank the Junta de Andalucía, MEC and IEM.

\section{References}

1.- D. J. Gross and F. Wilczek, Phys. Rev., D9 (1974) 980; H. Georgi and H. D. Politzer, Phys. Rev., D9 (1974) 416; I. Hinchliffe and C. H. Llewellyn Smith, Nucl. Phys., B128 (1977) 93; A. De Rújula, H. Georgi and H. D. Politzer, Ann. Phys. (NY), 103 (1977) 315.

2.- $\quad$ A. González-Arroyo, C. López and F. J. Ynduráin, Nucl. Phys., B153 (1979) 161; B159 (1979) $512 ; B 174,(1980) 474$.

3.- $\quad$ E. G. Floratos, D. A. Ross and C. T. Sachrajda, Nucl. Phys., B129 (1978) 66; (E) B139 (1978) 545.

4.- $\quad$ W. A. Bardeen et Al., Phys. Rev., D18 (1978) 3998.

5.- D. J. Gross, Phys. Rev. Lett., 32 (1974) 1071.

6.- W. Furmanski and R. Petronzio, Phys. Lett., 97B (1980) 437.

7.- $\quad$ K. Adel, F. Barreiro and F. J. Ynduráin, Nucl. Phys., B495 (1997) 221.

8.- W. L. van Neerven and E. B. ZiJlstra, Phys. Lett., B272 (1991) 127 and 476; ibid B273 (1991) 476; Nucl. Phys. B383 (1992) 525.

9.- $\quad$ S. A. Larin et Al., Nucl. Phys., B427 (1994) 41 and B492 (1997), 338.

10.- A. L. Kataev, G. Parente and A. V. Sidorov, JinR E2-98-265 (1998).

11.- $\quad$ F. J. Ynduráin, Phys. Lett., 74B (1978) 68.

12.- F. J. YndurÁin, The Theory of Quark and Gluon Interactions, 3rd. Ed., Springer-Verlag, New York, 1999.

13.- $\quad$ S. A. Larin, T. van Ritbergen and J. A. M. Vermaseren, B438, 1995 (278) ; K. G. Chetyrkin ET AL., Phys. Rev. Lett., 79 (1997) 2184.

14.- F. J. YndurÁin, The Moment Problem and Applications, Int'l School on Padé Approximants, The British Institute of Physics, 1973.

15.- $\quad$ A. D. Martin et AL., Eur. Phys. J., C4 (1998) 463.

16.- $\quad$ L. W. Whitlow et Al., Phys. Lett., B282 (1992) 475.

17.- $\quad$ A. Benvenuti et AL., Phys. Lett., B223 (1989) 485.

18.- M. R. Adams et Al., Phys. Rev., D54 (1996) 3006.

19.- $\quad$ M. Derrick et Al., Z. Phys., C72 (1996) 399 and S. Aid et Al., Nucl. Phys., B470 (1996) 3.

20.- F. James, CERN Program Library Long Writeup D506, version 94.1 (1994).

21.- O. Nachtmann, Nucl. Phys., B63 (1973) 237; H. Georgi and H. D. Politzer, Phys. Rev., D14 (1976) 1829.

22.- A. Pineda and F. J. Ynduráin, Phys. Rev., D58 (1998) 094022 and CERN-TH 98-402 (hepph/9812371).

23.- $\quad$ S. Bethke, Nucl. Phys. Proc. Suppl., B64 (1998) 54. 